Original article

\title{
Smooth muscle cells in human atherosclerosis: Proteomic profiling reveals differences in expression of Annexin A1 and mitochondrial proteins in carotid disease
}

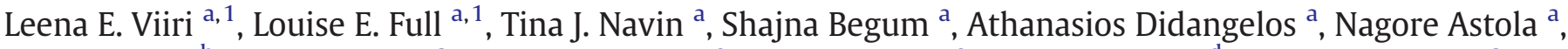 \\ Rolf K. Berge ${ }^{b}$, Ilkka Seppälä ${ }^{c}$, Joseph Shalhoub ${ }^{\text {, }}$, Ian J. Franklin ${ }^{\text {e }}$, Mauro Perretti ${ }^{\mathrm{d}}$, Terho Lehtimäki ${ }^{\mathrm{c}}$, \\ Alun H. Davies ${ }^{\mathrm{e}}$, Robin Wait ${ }^{\mathrm{a}}$, Claudia Monaco ${ }^{\mathrm{a}, *}$
}

\footnotetext{
a Kennedy Institute of Rheumatology, Kennedy Institute, Nuffield Department of Orthopedics, Rheumatology and Musculoskeletal Sciences, University of Oxford, 65 Aspenlea Road, W6 8LH London, United Kingdom

${ }^{\mathrm{b}}$ Institute of Medicine, Haukeland University Hospital, University of Bergen, $N 5021$ Bergen, Norway

c Department of Clinical Chemistry, Tampere University Hospital and School of Medicine, University of Tampere, Finland

d William Harvey Research Institute, Queen Mary University of London, London, United Kingdom

e Imperial Vascular Unit, Surgery and Cancer, Faculty of Medicine, Imperial College London, United Kingdom
}

\section{A R T I C L E I N F O}

\section{Article history:}

Received 1 May 2012

Received in revised form 1 November 2012

Accepted 3 November 2012

Available online 12 November 2012

\section{Keywords:}

Atherosclerosis

Carotid plaque

Smooth muscle cell

Annexin A1

Inflammation

Mitochondria

\begin{abstract}
A B S T R A C T
Smooth muscle cells (SMC) contribute to the development and stability of atherosclerotic lesions. The molecular mechanisms that mediate their properties are incompletely defined. We employed proteomics and in vitro functional assays to identify the unique characteristics of intimal SMC isolated from human carotid endarterectomy specimens and medial SMC from thoracic aortas and carotids. We verified our findings in the Tampere Vascular Study. Human atheroma-derived SMC exhibit decreased expression of mitochondrial proteins ATP Synthase subunit-beta and Aldehyde dehydrogenase 2, and decreased mitochondrial activity when compared to control SMC. Moreover, a comparison between plaque-derived SMC isolated from patients with or without recent acute cerebrovascular symptoms uncovered an increase in Annexin A1, an endogenous anti-inflammatory protein, in the asymptomatic group. The deletion of Annexin A1 or the blockade of its signaling in SMC resulted in increased cytokine production at baseline and after stimulation with the pro-inflammatory cytokine Tumor Necrosis Factor $\alpha$. In summary, our proteomics and biochemical analysis revealed mitochondrial damage in human plaque-derived SMC as well as a role of Annexin A1 in reducing the production of pro-inflammatory mediators in SMC.
\end{abstract}

(c) 2012 Elsevier Ltd. All rights reserved.

\section{Introduction}

The seminal work of Campbell and Campbell demonstrated that smooth muscle cells (SMC) undergo 'phenotypic modulation' from a quiescent to a synthetic state within the atherosclerotic intima to accommodate an increased synthesis of extracellular proteins [1] that strengthen the fibrous cap [2]. Thus, the survival of SMC is essential to plaque stability [2]. Classical studies by Bennett and Schwartz have shown that cultured SMC isolated from atherosclerotic lesions display decreased rates of proliferation and increased senescence and apoptosis compared to medial SMC [3]. Importantly, apoptosis of SMC led to increased inflammatory cell infiltration, suggesting that the survival of SMC may modulate inflammation within atherosclerotic plaques [4]. The molecular mechanisms through which SMC can modulate the inflammatory response are currently unknown.

\footnotetext{
* Corresponding author at: Kennedy Institute, Nuffield Department of Orthopedics, Rheumatology and Musculoskeletal Sciences, University of Oxford, 65 Aspenlea Road, W6 8LH London, United Kingdom. Tel.: +44 208383 4765; fax: + 442083834499.

E-mail address: claudia.monaco@kennedy.ox.ac.uk (C. Monaco).

1 These authors contributed equally to this manuscript.
}

Proteomics approaches provide an unbiased assessment of the protein components of diseased tissues. Proteomics analyses in carotid tissue have generated biomarkers for cardiovascular events, such as osteopontin [5]. Only transcriptomic approaches have so far been applied to SMC from human atherosclerotic lesions [6,7], while no study has thus far compared the proteome of SMC derived from symptomatic or asymptomatic carotid disease and control vascular tissue.

In order to identify qualitative protein changes in SMC in established human atherosclerosis, we performed a proteomics characterization of SMC derived from carotid artery stenosis and control vascular tissue. We herein describe that Annexin A1 (ANXA1) levels in SMC are modulated in carotid disease and that ANXA1 signaling blockade or its deletion increases cytokine and chemokine production by SMC. We also show that mitochondrial damage is a distinctive feature of SMC isolated from human atherosclerotic lesions.

\section{Materials and methods}

An expanded Materials and methods section is available in the Supplementary data. 


\subsection{Study population}

For the proteomics profiling carotid plaques were obtained from 10 patients undergoing carotid endarterectomy at the Imperial Vascular Unit, London. Ethical approval was obtained (Riverside Research Ethics Committee 2989) and all patients gave their written informed consent. A patient was defined symptomatic if she/he had experienced, within the last 6 months, focal neurological symptoms pertaining to the ipsilateral carotid territory (stroke, transient ischemic attack or amaurosis fugax). Six out of 10 patients recruited matched these criteria (symptomatic group). The remaining 4 patients included in the study had carotid stenosis but did not experience acute symptoms (asymptomatic group). Each clinical case was presented in full at a specialist regional neuro-vascular multi-disciplinary team meeting where the index carotid stenosis was confirmed as responsible for the symptoms by highly experienced vascular clinicians, surgeons and radiologists on the basis of neurovascular imaging and exclusion of alternative embolic sources, including atrial fibrillation. All patients were on statins to avoid differences due to disease modulation. Patient characteristics are presented in Supplemental Table SI.

\subsection{Genome wide expression studies in the Tampere Vascular Study}

In order to eliminate the possibility that changes in the molecules of interest were due to the culture conditions, we verified their gene expression in the Tampere Vascular Study cohort [8]. Patient characteristics relative to the Tampere Vascular Study are presented in Supplemental Table SII. An extended methodology section on the Tampere Vascular Study is included in the online supplement.

\subsection{SMC isolation and culture}

Carotid endarterectomies were enzymatically digested immediately after collection and the cell suspension underwent negative selection with magnetic beads (Miltenyi) in order to obtain a pure population of plaque-derived intimal SMC (AthSMC) as previously described [9] and as detailed in the online supplement. Attempts to isolate SMC from the small medial segments attached to carotid endarterectomy specimens were unsuccessful in the majority of cases and did not result in enough cells for proteomics studies. However, we were able to isolate enough medial smooth muscle cells from 3 carotid specimens for selected validation studies. Human medial thoracic aorta-derived SMC (AoSMC) from 6 donors (mean age 31.5 years) were purchased from PromoCell (GmBH Heidelberg, Germany) and used as controls for the proteomics study. SMC were consistently used at passage 3 in all experiments. SMC were serum starved for 48 hours and were subsequently cultured in the presence or absence of Tumor Necrosis Factor $\alpha$ (TNF $\alpha ; 10 \mathrm{ng} / \mathrm{mL}$ ) for 24 hours.

\subsection{Proteomics}

Full description of the 2D electrophoresis and Mass Spectrometry methodology is included in the online supplement.

\subsection{Validation experiments}

Differential expression of proteins between the different groups was validated by immunoblotting and real-time quantitative PCR. Changes in mitochondrial protein expression were further validated functionally by quantifying mitochondrial dysfunction and cell apoptosis via citrate synthase and cytochrome c assay, respectively. Changes in proteasome activity in AthSMC and AoSMC were also assessed.

\subsection{Annexin A1 blockade}

ANXA1 signals through the $\mathrm{N}$-formyl peptide receptor 2 (FPR2), which has a structural homologue FPR1. To assess whether inhibition of binding of ANXA1 to its receptors would affect the response to TNF $\alpha$ of human AoSMC, we used two different ANXA1 receptor blockers, cyclosporin $\mathrm{H}$ (FPR1 antagonist) and $\mathrm{WRW}_{4}$ (FPR2 antagonist). AoSMC were serum-starved 24 hours prior to incubation with either vehicle, cyclosporin $\mathrm{H}(\mathrm{CsH}, 10 \mu \mathrm{M})$ or $\mathrm{WRW}_{4}(10 \mu \mathrm{M})$ for 30 minutes followed by incubation with or without TNF $\alpha(10 \mathrm{ng} / \mu \mathrm{l})$. The concentration of IL-6, MCP-1/CCL2, Fractalkine, IP-10/CXCL10, RANTES/CCL5 in cell culture supernatants was measured after 24 hours using a commercially available Luminex assay (Human cytokine/Chemokine Assay, Millipore).

\subsection{Isolation and culture of Anxa1 ${ }^{-/-}$aortic SMC}

We isolated SMC from the thoracic aortas of Anxa1 ${ }^{-/-}[10]$ and wild type mice (see online supplement for further details) by enzymatic digestion. SMC from at least 4 mice were pooled for experiment, serum starved 24 hours and subsequently stimulated with murine TNF $\alpha(10 \mathrm{ng} / \mathrm{ml})$ or left unstimulated. IL-6 concentration in cell culture supernatants was measured after 24 hours using commercially available ELISA (DuoSet ELISA, R\&D Systems). Furthermore, cells were lysed for RNA and gene expression levels of IL-6 and CCL2 were studied using individual TaqMan assays (LifeTechnologies).

\subsection{Statistical analyses}

Data are shown as mean \pm SEM. Statistical analysis of 2D gel data employed the statistics module of Progenesis Samespots (Nonlinear Dynamics, UK). Other analyses were performed using GraphPad Prism version 5.0 for Windows (GraphPad Software, San Diego, California, USA). One-way ANOVA, Student's t-test or repeated measures 2-way ANOVA were used as appropriate. All tests were 2-tailed. Statistical significance was considered for $P<0.05$. GenePattern software (version 3 ) was used to generate a heatmap representing the relative difference of the $2 \mathrm{D}$ spot volume for each identified protein [11]. The principal component analysis (PCA), using an orthogonal transformation to perform eigenvalue decomposition of the data into two uncorrelated variables was also computed using the GenePattern software.

\section{Results}

\subsection{AthSMC show evidence of phenotypic modulation}

Both AthSMC and AoSMC appeared spindle-shaped and displayed the characteristic hill and valley pattern at confluence (Fig. S1A). Evaluation of the contractile markers smooth muscle $\alpha$-actin ( $\alpha \mathrm{SMA})$ and smooth muscle myosin heavy chain (SM-MHC) by PCR, immunofluorescence and immunoblotting demonstrated a decrease in $\alpha \mathrm{SMA}$ and SM-MHC expression in AthSMC compared with AoSMC (Fig. S1B-D).

Eighty-four spots on the 2D gels were identified by Mass Spectrometry (Table SIII). The 2D gel analysis with Samespots software demonstrated that thirteen spots out of 84 were differentially expressed between the AthSMC $(n=10)$ and AoSMC ( $n=6$; Table SIV). The first notable change in AthSMC compared to AoSMC was the reduction in cytoskeletal proteins actin and vimentin. Actin was identified as a train of spots (19-23 in Fig. S1E and Table SIII), with a molecular mass of approximately $40 \mathrm{kDa}$ and isoelectric points between 5.3 and 6.0, which would be due to post-translational modifications, such as acetylation. There is $94 \%$ sequence homology between $\beta$-actin and $\alpha$ SMA. All but one (GYSFTTTAERE- $\beta$-actin specific) of the peptides detected belonged to both sequences. Thus, the identified actin could be either $\beta$-actin or $\alpha S M A$. Four of the actin spots were significantly decreased in volume in AthSMC compared to AoSMC (spot 19, 20 and 21, $P<0.05$; spot 23, $P<0.01$; Fig. S1E and Table SIV). In keeping with this finding, four 
spots (Fig. S2 and Table SIII) were identified as vimentin, the major constituent of the intermediate filaments of the cytoskeleton. One of these spots ( spot 83) was decreased in AthSMC compared to AoSMC $(P<0.01$; Table SIV), in accordance with previous literature showing a decrease in vimentin in atherosclerotic intima [12].

Inflammation is one of the factors distinguishing the plaque environment from the normal arterial wall. We therefore investigated TNF $\alpha$ as a model pro-inflammatory stimulus on the SMC proteome. After TNF $\alpha$ stimulation, two actin spots were statistically significantly decreased in both AthSMC and AoSMC (Fig. S3A). Accordingly, the actin-associated protein F-actin capping subunit alpha (CAPZA1) was decreased in TNF $\alpha$-stimulated AoSMC by 27\% (Fig. S3D).

3.2. Mitochondrial damage and over-oxidation of peroxiredoxins are features of AthSMC

Two key mitochondrial proteins were differentially expressed in AthSMC vs. AoSMC (Fig. 1A) in the 2D gel analysis. The expression of ATP synthase beta subunit (ATP5B), which is part of the F1 catalytic core of ATP Synthase (complex V) in the electron transport chain was lower in AthSMC than in AoSMC $(P<0.001$; Fig. 1A). Similarly, aldehyde dehydrogenase 2 (ALDH2), important in the detoxification of reactive aldehydes, was decreased in AthSMC vs. AoSMC $(P<0.05$; Fig. 1A). The significant decrease of ATPB5 and ALDH2 proteins in AthSMC vs. AoSMC was confirmed by Immunoblotting $(P<0.01$; Figs. 1B-C). Quantitative Real-Time PCR also showed a statistically significant decrease of ALDH2 in AthSMC vs. AoSMC ( $P<0.001$, Fig. 1D), albeit ATP5B gene expression was not statistically significant (data not shown). Subsequently, we compared the expression of the mitochondrial proteins ATP5B and ALDH2 in medial and intimal AthSMC to exclude the possibility that the differences observed were due to sampling of different arterial sites. We were able to confirm a reduced expression of both genes in diseased intimal compared to the medial AthSMC ( $P<0.05$, Fig. 1E), indicating that the differences in mitochondrial proteins could not be explained by vascular site or embryological origins of the SMC.

Next we sought to assess whether the decrease of ATP5B and ALDH2 in AthSMC was the result of a decrease in mitochondrial mass. Since citrate synthase is exclusively localized in mitochondria, its activity is used as a marker for mitochondrial mass $[13,14]$. AthSMC had significantly lower citrate synthase activity compared with AoSMC $(P<0.0001$; Fig. 1F). To assess whether the change in mitochondrial mass in AthSMC was secondary to apoptosis, we quantified the cytosolic levels of cytochrome $\mathrm{c}$, a protein associated with the inner membrane of the mitochondrion [15] and released into the cytosol in response to apoptotic
A

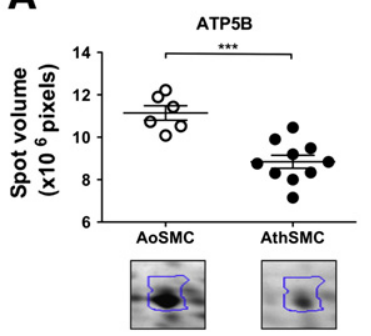

B

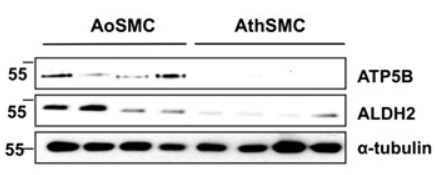

C

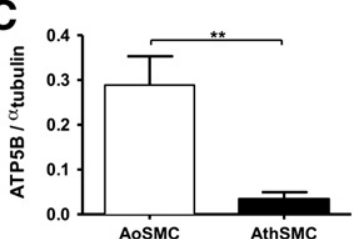

E

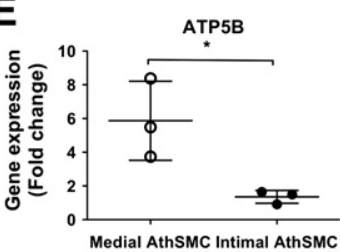

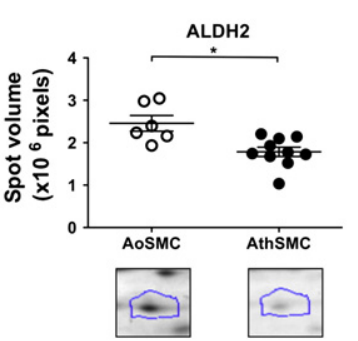
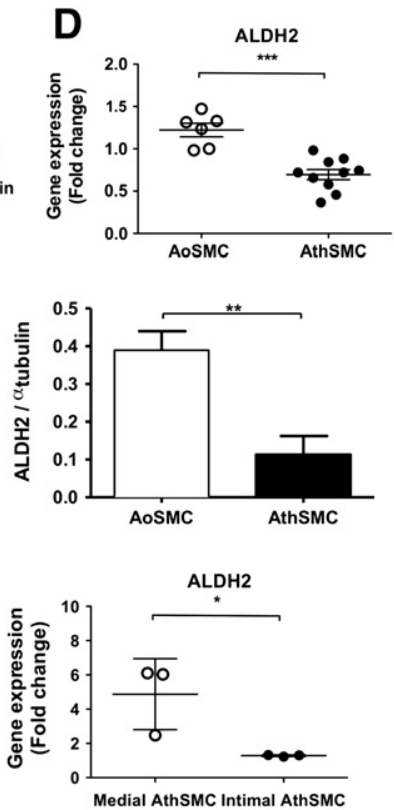

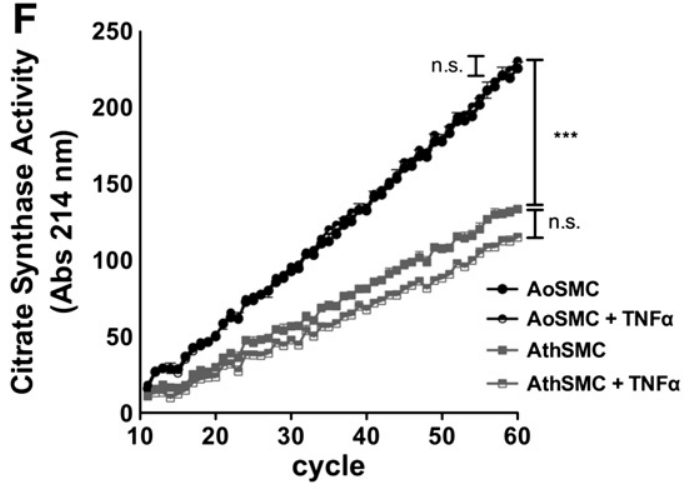

G
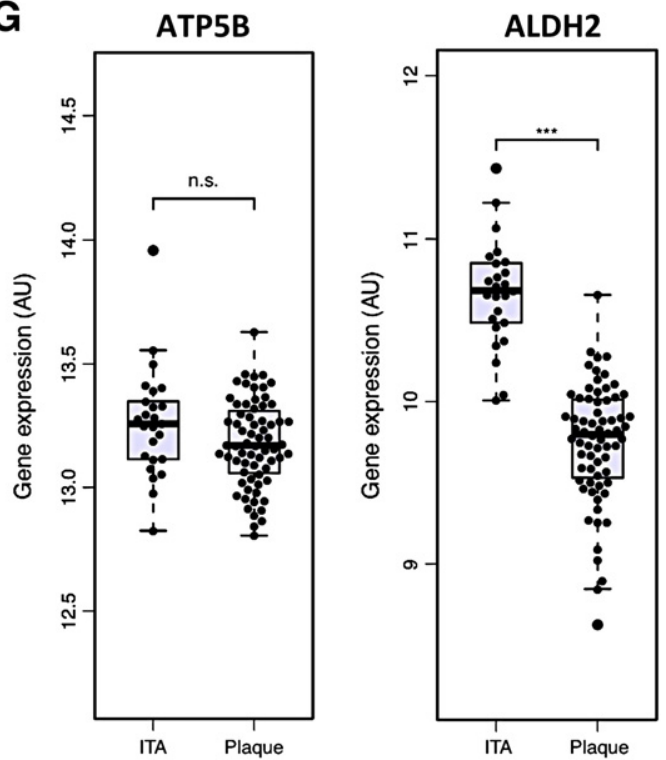

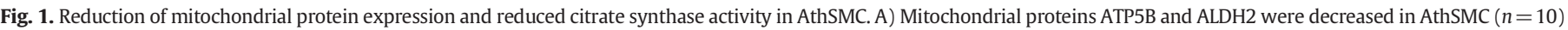

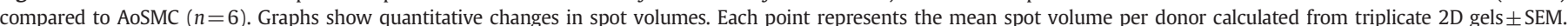

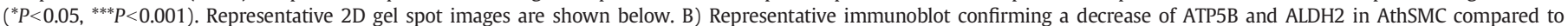

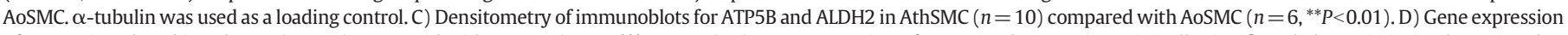

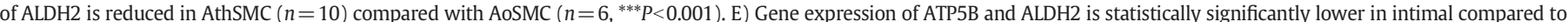

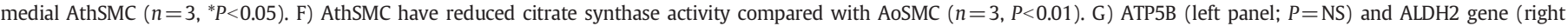

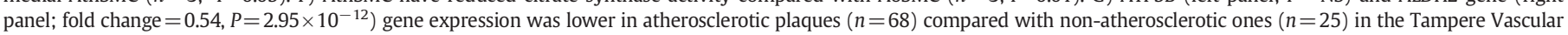
Study (AU = arbitrary unit). 
signals [16]. Cytosolic cytochrome c concentrations did not differ between AthSMC and AoSMC ( $P=$ NS; Fig. S4), indicating that a decrease in mitochondrial mass -but not apoptosis - is responsible for the decrease in ATP5B and ALDH2 in AthSMC. Moreover, gene expression studies in the Tampere Vascular Study revealed lower ALDH2 $(P=$ $2.95 \times 10^{-12}$, Fig. $\left.1 G\right)$ and ATP5B ( $P=$ NS; Fig. $\left.1 G\right)$ gene expression in atherosclerotic plaques compared with non-atherosclerotic ones, suggesting that changes in the expression of mitochondrial proteins are not only related to the culture conditions but are present in undissociated tissues.

We have previously shown that mice transgenic for human TNF $\alpha$ have reduced mitochondrial function and fatty acid oxidation [17]. Thus we evaluated whether TNF $\alpha$ might modulate the expression of the mitochondrial proteins ATP5B and ALDH2. TNF $\alpha$-stimulation of AthSMC caused a 15\% decrease in ATP5B and 32\% decrease in ALDH2 compared to unstimulated AthSMC (Fig. S3B). However, the difference in citrate synthase activity between TNF $\alpha$ stimulated and unstimulated SMC was not statistically significant (Fig. 1F).

An important pathway for mitochondrial dysfunction is the exposure to excessive oxidative stress. Two spots were identified as the antioxidant protein peroxiredoxin-4 (PRDX4) respectively in the oxidized and reduced form. The over-oxidized form of PRDX4 was increased in AthSMC compared to AoSMC $(P<0.05$; Fig. S5A-C; Table SIV $)$. As there was no corresponding reduction in the reduced form (Fig. S5C), this finding might indicate that AthSMC have been exposed to increased oxidative stress resulting in an overall increase in expression and oxidation of PRDX4. In support of this finding, analyses in the Tampere Vascular Study showed an increase in PRDX4 gene expression in atherosclerotic plaques compared with the non-atherosclerotic controls $\left(P=1.12 \times 10^{-11}\right.$; Fig. S5D). The expression levels of ATP5B, ALDH2 and PRDX4 in the Tampere Vascular Study did not vary in plaques with different Stary Classification types (Fig. S6), with the presence of intraplaque hemorrhage or thrombus (Fig. S7), or patient symptoms (Fig. S8). Moreover, no differences were found when patients were grouped according to risk factors (Table SV), suggesting that the differences in such genes are not restricted to a specific risk factor or plaque development stage.

3.3. Differences in the proteome of AthSMC derived from symptomatic and asymptomatic patients include changes in contractile proteins and Annexin A1

We next investigated whether the symptomatic status of patients influenced the properties of their AthSMC. In a comparison between 2D gel images with the Samespots software between AoSMC $(n=6)$, asymptomatic $(n=4)$ and symptomatic $(n=6)$ AthSMC, 28 spots were differentially expressed and positively identified by mass spectrometry. The relative difference in spot volume for each identified protein is shown as a heat map in Fig. 2A and in Table SVI. Interestingly, eigenvalue decomposition of the data into two uncorrelated principal components using PCA (94\% of the data variance) indicated that the protein profile of symptomatic patients with carotid disease was distinctively different from the protein profile from both asymptomatic patients and AoSMC (Fig. 2B). Thirteen of the spots were differently expressed between AthSMC from symptomatic $(n=6)$ and asymptomatic $(n=4)$ patients (Table SVII).

Actin expression was increased by approximately $30-50 \%$ in AthSMC derived from asymptomatic patients compared to symptomatic patients $(P<0.05$; Fig. $3 \mathrm{~A})$. A decrease of contractile proteins $\alpha S M A$ and SM-MHC in AthSMC of symptomatic patients was also confirmed via PCR (Fig. S9). In addition, actin binding protein F-actin capping protein subunit alpha- 1 was decreased by $46 \%$ in symptomatic AthSMC $(P<0.01$; Table SVII). Instead, the expression of oxidized PRDX4 and of the mitochondrial proteins ATP5B and ALDH2 did not differ between symptomatic and asymptomatic SMC (Figs. 3B-C).

Of the differentially expressed proteins, Annexin 1 (ANXA1) was of particular interest, due to its potent anti-inflammatory properties.
ANXA1 was increased in asymptomatic SMC compared to symptomatic SMC $(P<0.05$; Fig. $4 \mathrm{~A})$. This observation was confirmed at the gene level $(P<0.05$; Fig. $4 \mathrm{~B})$ and via immunoblotting $(P<0.01$; Figs. $4 C-D)$. Moreover, in the Tampere Vascular study, ANXA1 gene expression was significantly higher in atherosclerotic plaques compared with non-atherosclerotic controls $\left(P=2.95 \times 10^{-5}\right.$; Fig. 4E). The expression levels of ANXA1 in the Tampere Vascular Study did not vary in plaques with different Stary Classification types (Fig. S6), or with the presence of intraplaque hemorrhage or thrombus (Fig. S7). Moreover, no differences in its expression were found when patients were grouped according to risk factors (Table SV), suggesting that the differences in ANXA1 are not simply a reflection of risk factors or plaque development stage. Only 2 patients in the Tampere Vascular Study were asymptomatic, but they had higher levels of ANXA1, in accordance with previous literature [18].

\subsection{Blockade of Annexin A1 signaling or Annexin A1 deletion increases cytokine and chemokine production in vascular SMC}

We next investigated the biological significance of ANXA1 in SMC behavior. ANXA1 signals through FPR2 and its structural homologue FPR1. We cultured AoSMC in the presence or absence of two selective FPR blockers, cyclosporin H (FPR1 antagonist) and WRW4 (FPR2 antagonist) to assess whether inhibition of the binding of ANXA1 to its receptors would affect the TNF $\alpha$ response of human SMC. We observed a significant increase in IL-6 and Fractalkine release at baseline and upon TNF $\alpha$ stimulation in the presence of both blockers $(P<0.001$; Fig. 5A). No significant changes were observed in the other mediators studied.

Finally, SMC isolated from thoracic aortas of Anxa1 $1^{-1-}$ mice displayed an increased expression of IL- 6 and CCL2 when compared to SMC isolated from thoracic aortas of WT mice both at baseline and upon stimulation with TNF $\alpha(P<0.001$; Fig. 5B). Similar changes were observed with IL-6 release (Fig. $5 C$ ).

\section{Discussion}

SMC are important arbiters of the outcome of atherosclerotic plaque formation. Our study is the first to characterize the proteome of SMC derived from human atherosclerotic lesions (AthSMC), and it reveals that AthSMC exhibit signs of exposure to oxidative stress and mitochondrial damage when compared to control SMC (AoSMC). We also show that SMC isolated from asymptomatic patients are qualitatively different from SMC isolated from symptomatic patients, as they have an increased expression of the potent anti-inflammatory protein annexin-A1 (ANXA1). Loss of ANXA1 or blockade of ANXA1 signaling was associated with increased production of IL-6, CCL2 and fractalkine in vascular SMC, suggesting that ANXA1 expression in SMC may modulate the inflammatory response.

Mitochondrial dysfunction is linked to insulin resistance and atherosclerosis [19]. We found a significant decrease in expression of two key mitochondrial proteins in AthSMC compared to AoSMC. This decrease was independently verified in the extended cohort of the Tampere Vascular Study. The reduction in ATP5B and ALDH2 in AthSMC was associated with a decrease in mitochondrial abundance as assessed via the citrate synthase assay, indicating that loss of ATP5B and ALDH2 may be a true indicator of the presence of mitochondrial damage. Concomitantly, we observed a compensatory increase in the expression of the glycolytic enzyme pyruvate kinase in AthSMC compared to AoSMC (Table SIV). This is consistent with the increased levels of alpha-enolase and triose phosphate isomerase reported in murine atherosclerotic aortas [20], and with the possibility of damage to the electron transport chain, of which ATP5B is a key participator. The levels of ATP5B and ALDH2 in AthSMC decreased with TNF $\alpha$ stimulation but the difference was not reflected in the citrate synthase activity between the stimulated and unstimulated SMC, suggesting that a prolonged exposure to TNF $\alpha$ may be necessary to induce mitochondrial changes or that other mechanisms 

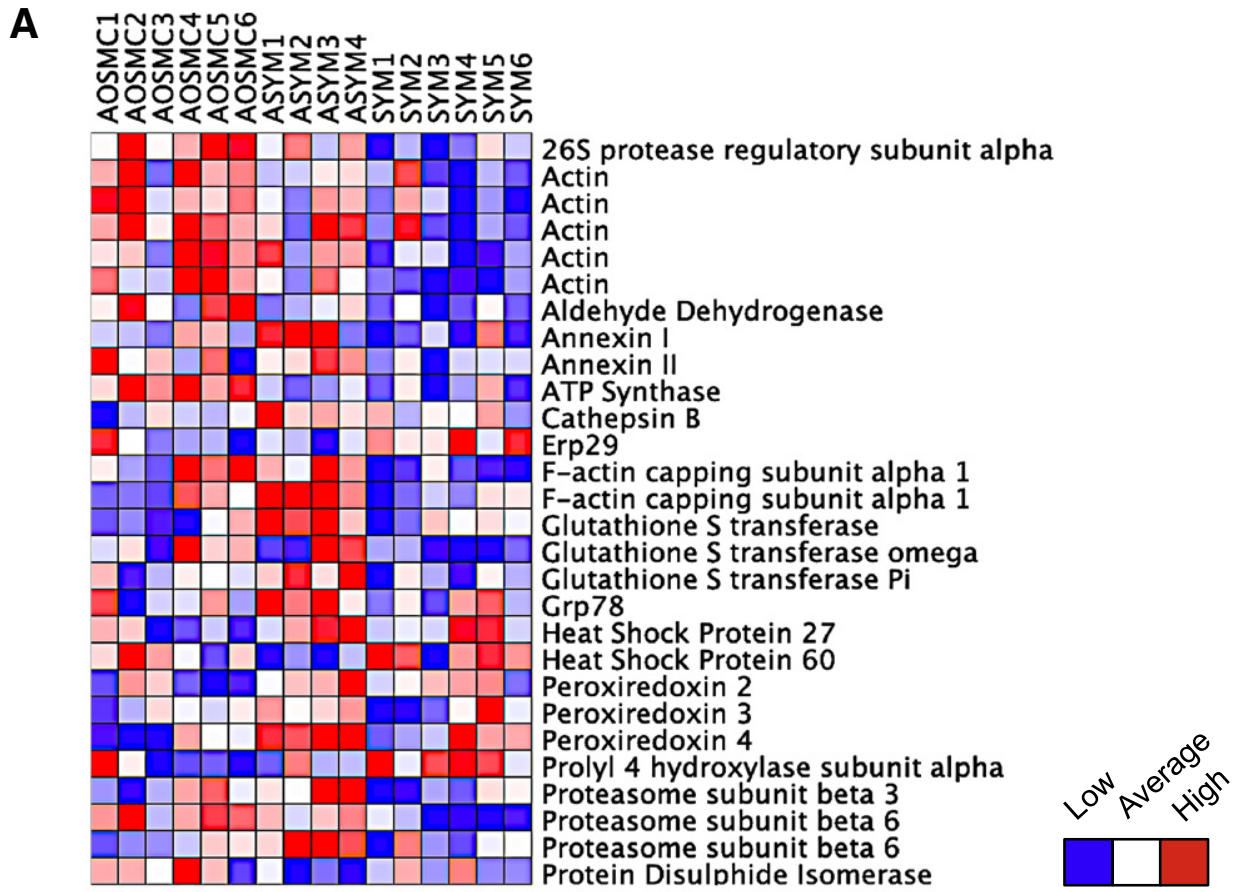

B

2D projection

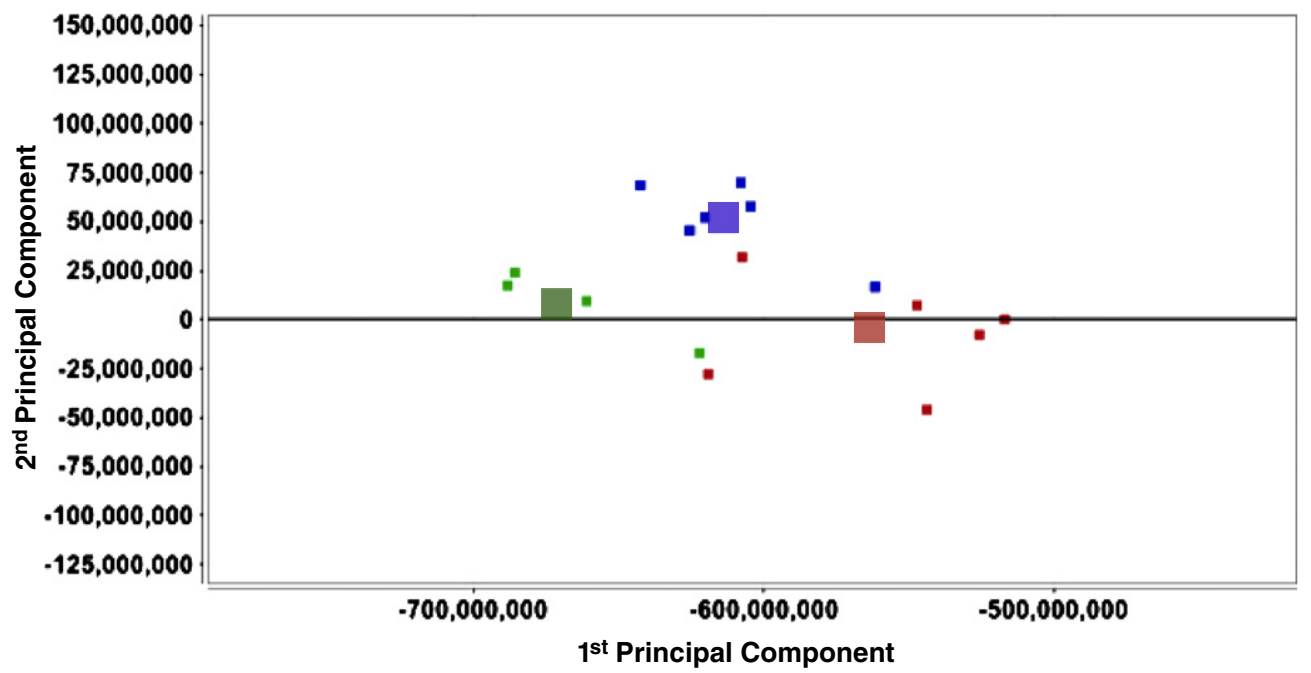

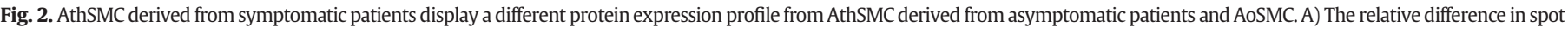

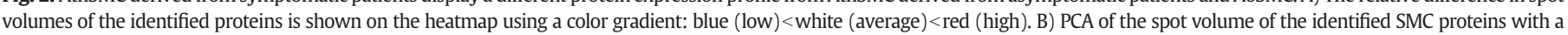

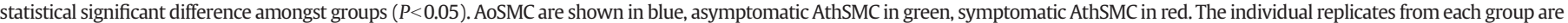
represented by the small rectangles, while the larger rectangles show the average value for the 2 principal components from each group.

might be involved in determining overall mitochondrial function. The identification of specific proteins ATP5B and ALDH2 as associated with conditions of mitochondrial damage in the atherosclerotic plaque raises hope of their suitability as future biomarkers of mitochondrial function in atherosclerosis and other conditions.

The causes of mitochondrial dysfunction in atherosclerosis are likely to be complex. Mitochondrial DNA is particular susceptible to DNA damage. Apolipoprotein $\mathrm{E}(\mathrm{ApoE})^{-/-}$mice deficient in the DNA repair enzyme ataxia telangiectasia mutated protein, had marked mitochondria dysfunction and accelerated atherosclerosis [19]. We were not able to detect the specific mitochondrial DNA deletion in human SMC (data not shown) suggesting other mechanisms may be at play in our setting. The release of mitochondria-associated proteins, such as cytochrome c, is a feature of apoptosis [16]. We did not detect variations in cytosolic release of cytochrome c between AthSMC and AoSMC, indicating that the differences observed in the levels of the mitochondrial proteins ATP5B and ALDH2 are not due to increased apoptosis of the AthSMC, but rather reflect mitochondrial damage. The concomitant finding of over-oxidation of peroxiredoxin- 4 in AthSMC compared with AoSMC confirm previous findings of increased over-oxidation of 1-Cys peroxiredoxin in murine atherosclerosis [20] and it might indicate that mitochondrial damage is carried out by increased oxidative stress. Of relevance, SMC are a major cellular source of reactive oxygen species in the vessel wall in models of arterial injury [21] and atherosclerosis [22]. With relevance to our set up, cultured SMC from Watanabe Heritable Hyperlipidemia (WHHL) rabbit aortas exhibited significantly greater basal levels of reactive oxygen species than SMC from control New Zealand rabbits [22]. Importantly, PRDX4 has been recently shown to exert a protective 
A

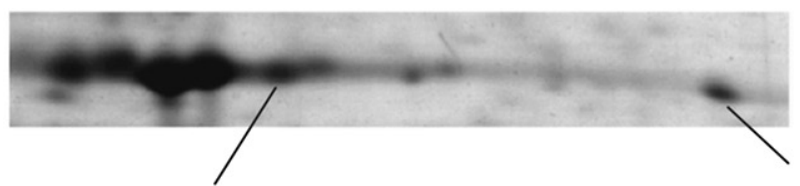

Actin 22

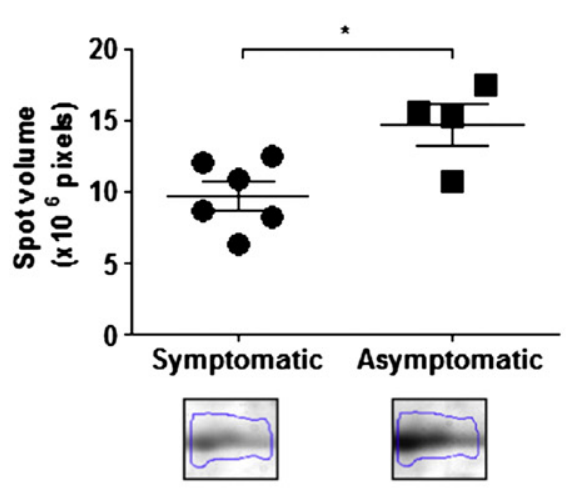

B

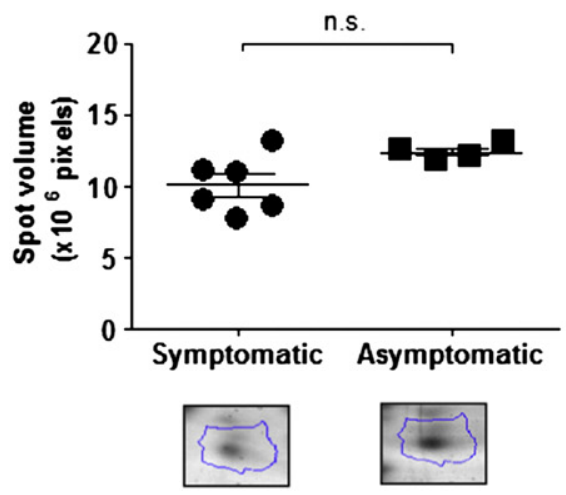

C

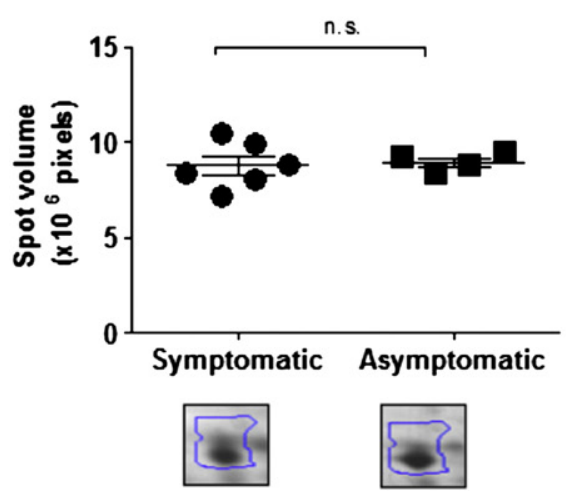

Actin 23

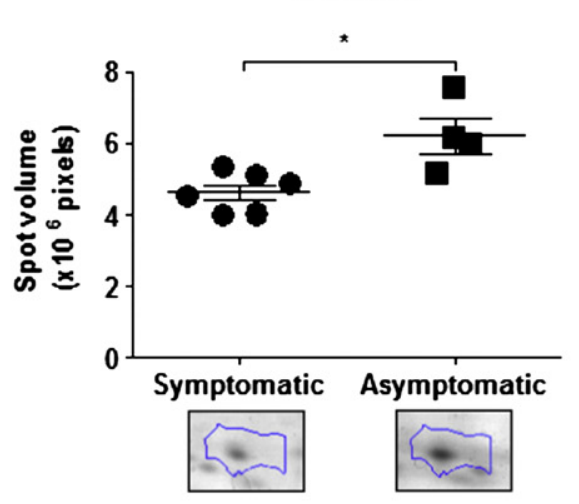

PRDX4 (reduced)
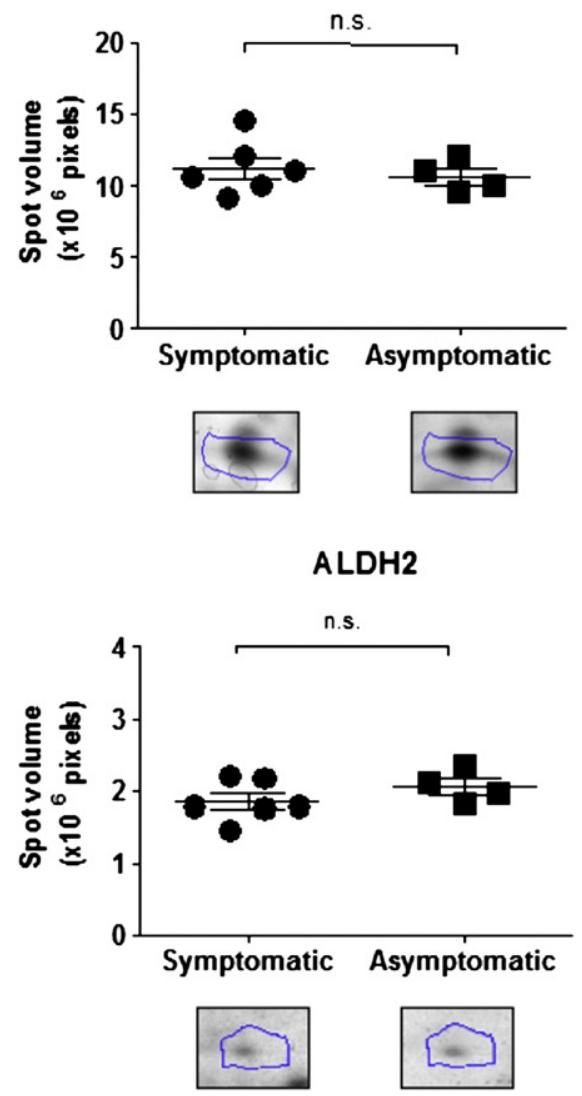

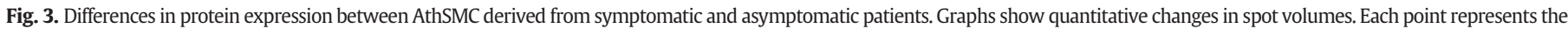

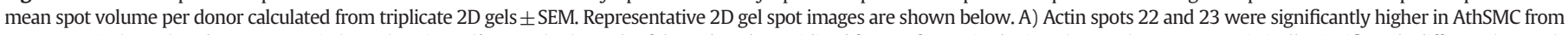

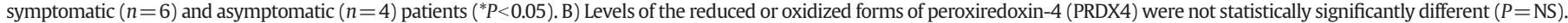
C) There was no difference in levels of ATP Synthase subunit-beta (ATP5B) or aldehyde dehydrogenase 2 (ALDH2; $P=$ NS).

action in atherosclerosis by suppressing oxidative damage [23]. In this study, lower levels of oxidative stress were detected in SMC in the plaques of $\mathrm{ApoE}^{-/-}$mice transgenic for human PRDX4 compared to ApoE $^{-/-}$mice [23].

ANXA1, a protein with potent anti-inflammatory effects [24], was expressed at higher levels in AthSMC isolated from asymptomatic compared to symptomatic patients. ANXA1 has previously been detected in atherosclerosis in cells staining with HAM56 antibody, which is reactive for macrophages and subpopulations of endothelial cells [25]. A recent study [18] reported increased ANXA1 protein in whole carotid plaques from asymptomatic vs. symptomatic patients, in keeping with our results. However, its functional role in atherosclerosis is yet unexplored. Anxa1 ${ }^{-/-}$mice have exacerbated inflammation [10]. We showed that when ANXA1 function is prevented via blockade of its receptors, SMC produce more inflammatory cytokines, an effect that is further promoted by TNF $\alpha$ stimulation. This supports the hypothesis that ANXA1 may 
A

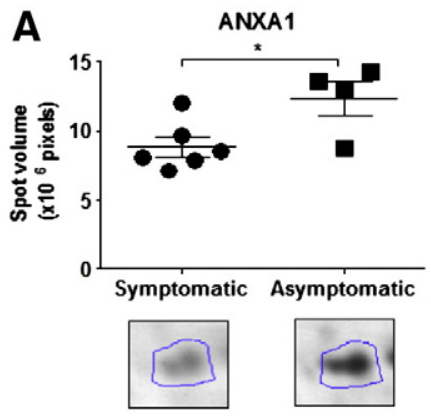

C

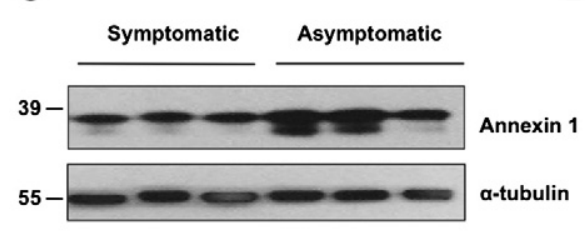

B

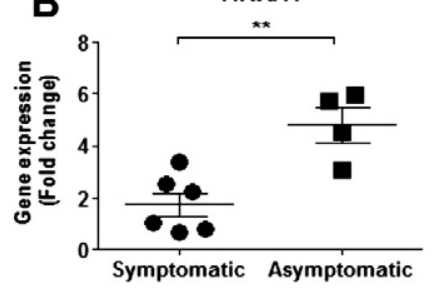

D

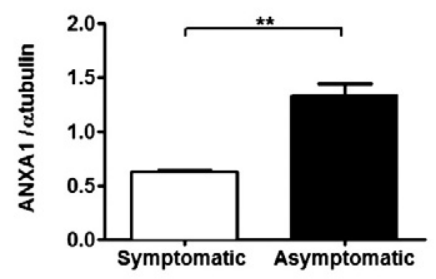

ANXA1

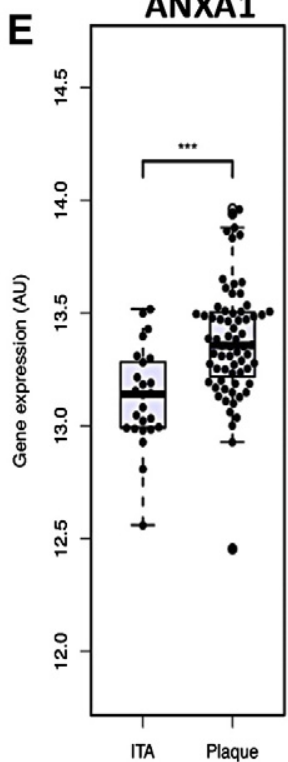

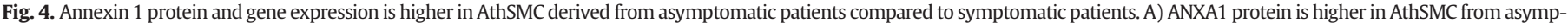

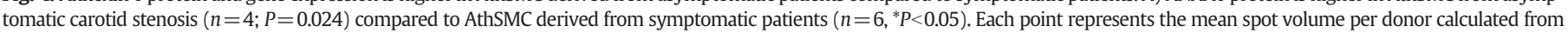

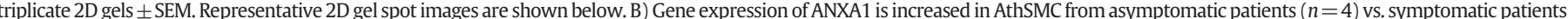

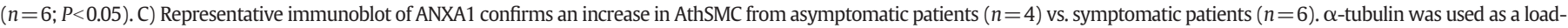

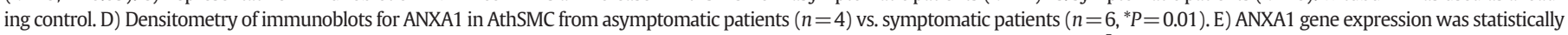
significantly higher in atherosclerotic plaques $(n=68)$ compared with non-atherosclerotic controls $\left(n=25 ; \mathrm{FC}=1.16, P=2.95 \times 10^{-5}\right)$ in the Tampere Vascular study.

exert anti-inflammatory effects by dampening the response to inflammatory stimuli (such as TNF $\alpha$ ) in SMC. Reduction of SMC numbers via induction of apoptosis has been experimentally shown to induce plaque inflammation [4], implicating either the production of pro-inflammatory mediators by apoptosis or the removal of anti-inflammatory mechanisms in this process. Further prospective and in vivo studies will be needed to ascertain a role for ANXA1 in preventing plaque inflammation. In accordance with our previous studies, we did not observe significant changes in ANXA1 after exposure to TNF $\alpha$ suggesting that other factors might be involved in the regulation of its expression [26,27].
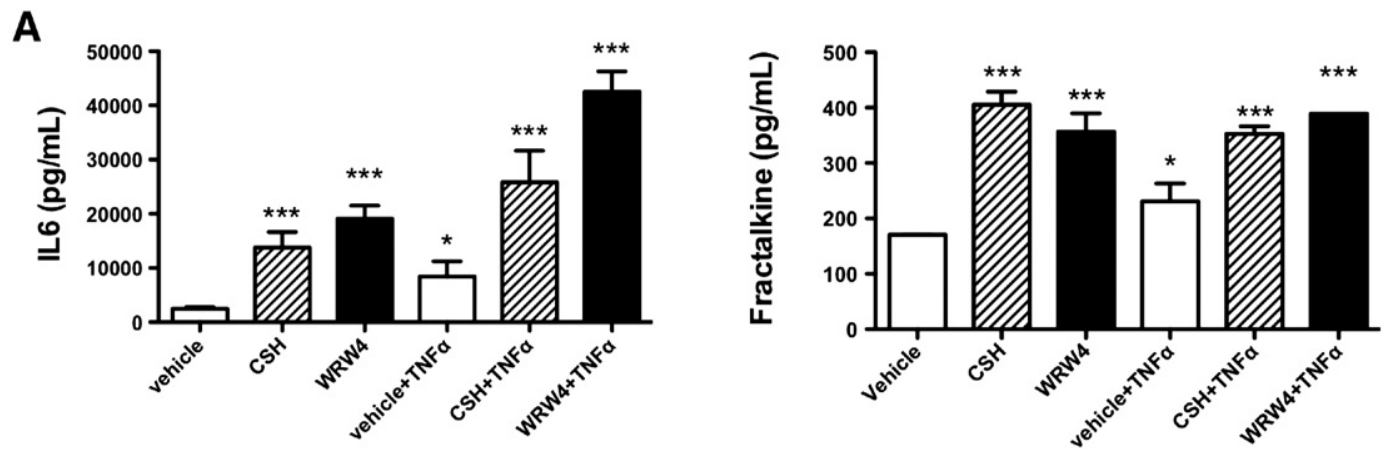

B

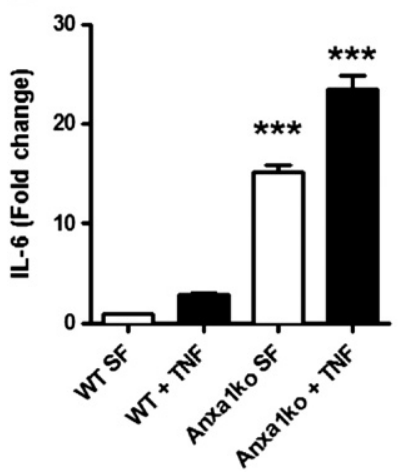

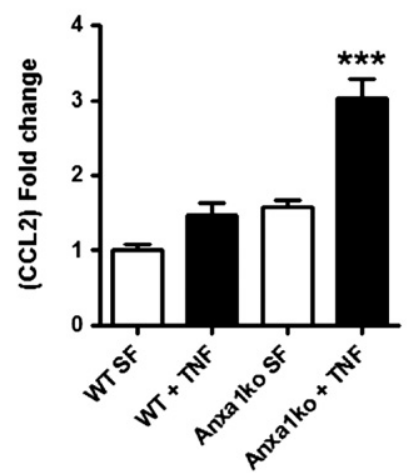

C

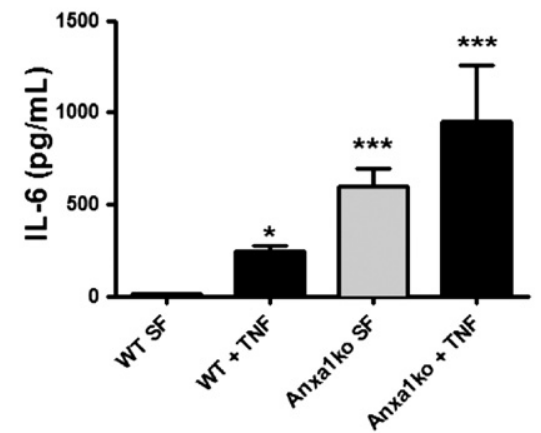

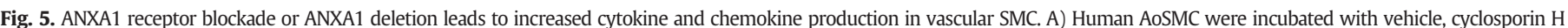

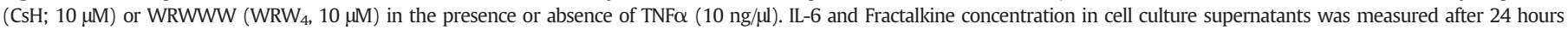

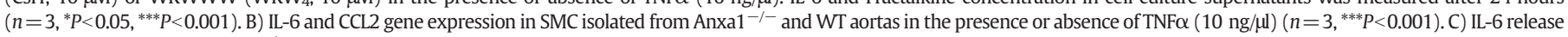
from SMC isolated from Anxa $1^{-/-}$and WT aortas in the presence or absence of TNF $\alpha(10 \mathrm{ng} / \mu \mathrm{l})\left(n=3,{ }^{*} P<0.05,{ }^{* * *} P<0.001\right)$. 
One limitation of our approach is the possibility that SMC lose their phenotype when extensively passaged. To minimize such effect we passaged cells no more than three times, and used both atheroma-derived and control SMC at the same early passage. Another challenge in this setting is the choice of control cells. Attempts to isolate AthSMC from medial segments were unsuccessful in $70 \%$ of the donors and did not result in enough cells for proteomics studies, limiting their use to selected validation studies. Carotid medial SMC are not commercially available. Thoracic aorta is much less prone to atherosclerosis than other available options, i.e. abdominal aorta or coronary arteries [28]. Albeit thoracic AoSMC have different embryonic origin [29], they are topographically the closest of all controls available and were thus considered to be the most suitable cells for our study. AthSMC were isolated using the same method (enzymatic digestion as opposed to explanting), grown in identical medium and used at the same cell passage as control medial AoSMC. We were able to reproduce finding of previous proteomics studies in atherosclerosis, e.g. increase in proteins related to oxidative stress and glucose metabolism [20]. In line with previous literature [30], we observed the down-regulation of cytoskeletal proteins in AthSMC compared to AoSMC. Our finding that TNF $\alpha$ stimulation decreases the expression of actin and of the actin-associated protein F-actin capping subunit also reproduces past observations [31]. The consistency of our findings with previous literature lends strong support to our experimental design. Moreover, Fig. 2 and Table SVI highlight that the proteome of AoSMC was remarkably similar to the SMC derived from asymptomatic carotid stenosis rather than the ones derived from symptomatic carotid stenosis. Finally, we were able to reproduce our findings in a comparison between carotid medial and intimal cells, underpinning the validity of our observations.

\section{Conclusions}

In established human atherosclerosis SMC acquire a distinct protein "signature" that suggests exhaustion of antioxidant mechanisms and mitochondrial damage. We identify biological differences between SMC extracted from patients with symptomatic and asymptomatic carotid disease, including the increased expression of Annexin A1 in SMC resident in asymptomatic carotid stenosis. Our findings underscore the protective role of SMC in advanced atherosclerosis and suggest Annexin A1 signaling as a novel mechanism through which SMC might dampen inflammation.

Supplementary data related to this article can be found online at http://dx.doi.org/10.1016/j.yjmcc.2012.11.002.

\section{Disclosure statement}

None declared.

\section{Acknowledgements}

This study was supported by European Commission (LSHM-CT2006-037400; IMMUNATH and FP7/2007-2013; 201668; AtheroRemo), The Graham-Dixon Charitable Trust, Yrjö Jahnsson Foundation, Medical Research Fund of Tampere University Hospital (9M048) and The Finnish Cardiovascular Foundation.

\section{References}

[1] Chamley-Campbell J, Campbell GR, Ross R. The smooth muscle cell in culture. Physiol Rev 1979;59:1-61.

[2] Libby P. Molecular bases of the acute coronary syndromes. Circulation 1995;91: 2844-50.
[3] Bennett MR, Evan GI, Schwartz SM. Apoptosis of human vascular smooth muscle cells derived from normal vessels and coronary atherosclerotic plaques. J Clin Invest 1995;95:2266-74

[4] Clarke MC, Figg N, Maguire JJ, Davenport AP, Goddard M, Littlewood TD, et al. Apoptosis of vascular smooth muscle cells induces features of plaque vulnerability in atherosclerosis. Nat Med 2006;12:1075-80.

[5] de Kleijn DPV, Moll FL, Hellings WE, Ozsarlak-Sozer G, de Bruin P, Doevendans PA, et al. Local atherosclerotic plaques are a source of prognostic biomarkers for adverse cardiovascular events. Arterioscler Thromb Vasc Biol 2010;30:612-9.

[6] Payeli SK, Latini R, Gebhard C, Patrignani A, Wagner U, Luscher TF, et al. Prothrombotic gene expression profile in vascular smooth muscle cells of human saphenous vein, but not internal mammary artery. Arterioscler Thromb Vasc Biol 2008:28:705-10.

[7] Zhang QJ, Goddard M, Shanahan C, Shapiro L, Bennett M. Differential gene expression in vascular smooth muscle cells in primary atherosclerosis and in stent stenosis in humans. Arterioscler Thromb Vasc Biol 2002;22:2030-6.

[8] Levula M, Oksala N, Airla N, Zeitlin R, Salenius JP, Jarvinen O, et al. Genes involved in systemic and arterial bed dependent atherosclerosis-Tampere Vascular study. PLoS One 2012;7:e33787.

[9] Cole JE, Navin TJ, Cross AJ, Goddard ME, Alexopoulou L, Mitra AT, et al. Unexpected protective role for Toll-like receptor 3 in the arterial wall. Proc Natl Acad Sci USA 2011;108:2372-7.

[10] Hannon R, Croxtall JD, Getting SJ, Roviezzo F, Yona S, Paul-Clark MJ, et al. Aberrant inflammation and resistance to glucocorticoids in annexin $1-/-$ mouse. FASEB J 2003; 17:253-5

[11] Reich M, Liefeld T, Gould J, Lerner J, Tamayo P, Mesirov JP. GenePattern 2.0. Nat Genet 2006;38:500-1.

[12] de la Cuesta F, Alvarez-Llamas G, Maroto AS, Donado A, Zubiri I, Posada M, et al. A proteomic focus on the alterations occurring at the human atherosclerotic coronary intima. Mol Cell Proteomics 2011;10 [M110 003517].

[13] Dimauro S, Tay S, Mancuso M. Mitochondrial encephalomyopathies: diagnostic approach. Ann N Y Acad Sci 2004;1011:217-31.

[14] Liu JT, Guo X, Ma WJ, Zhang YG, Xu P, Yao JF, et al. Mitochondrial function is altered in articular chondrocytes of an endemic osteoarthritis, Kashin-Beck disease. Osteoarthritis Cartilage 2010;18:1218-26.

[15] Gonzales DH, Neupert W. Biogenesis of mitochondrial c-type cytochromes. J Bioenerg Biomembr 1990;22:753-68.

[16] Liu X, Kim CN, Yang J, Jemmerson R, Wang X. Induction of apoptotic program in cell-free extracts: requirement for dATP and cytochrome c. Cell 1996;86:147-57.

[17] Glosli H, Gudbrandsen OA, Mullen AJ, Halvorsen B, Rost TH, Wergedahl H, et al. Downregulated expression of PPARalpha target genes, reduced fatty acid oxidation and altered fatty acid composition in the liver of mice transgenic for hTNFalpha. Biochim Biophys Acta 2005;1734:235-46.

[18] Cheuk BL, Cheng SW. Annexin A1 expression in atherosclerotic carotid plaques and its relationship with plaque characteristics. Eur J Vasc Endovasc Surg 2011;41:364-71.

[19] Mercer JR, Cheng KK, Figg N, Gorenne I, Mahmoudi M, Griffin J, et al. DNA damage links mitochondrial dysfunction to atherosclerosis and the metabolic syndrome. Circ Res 2010;107:1021-31.

[20] Mayr M, Chung YL, Mayr U, Yin X, Ly L, Troy H, et al. Proteomic and metabolomic analyses of atherosclerotic vessels from apolipoprotein E-deficient mice reveal alterations in inflammation, oxidative stress, and energy metabolism. Arterioscler Thromb Vasc Biol 2005;25:2135-42.

[21] Nunes GL, Robinson K, Kalynych A, King III SB, Sgoutas DS, Berk BC. Vitamins C and E inhibit O2- production in the pig coronary artery. Circulation 1997;96:3593-601.

[22] Miller Jr FJ, Gutterman DD, Rios CD, Heistad DD, Davidson BL. Superoxide production in vascular smooth muscle contributes to oxidative stress and impaired relaxation in atherosclerosis. Circ Res 1998;82:1298-305.

[23] Guo X, Yamada S, Tanimoto A, Ding Y, Wang KY, Shimajiri S, et al. Overexpression of peroxiredoxin 4 attenuates atherosclerosis in apolipoprotein e knockout mice. Antioxid Redox Signal 2012;17:1362-75.

[24] Perretti M, D'Acquisto F. Annexin A1 and glucocorticoids as effectors of the resolution of inflammation. Nat Rev Immunol 2009;9:62-70.

[25] Bagnato C, Thumar J, Mayya V, Hwang SI, Zebroski H, Claffey KP, et al. Proteomics analysis of human coronary atherosclerotic plaque: a feasibility study of direct tissue proteomics by liquid chromatography and tandem mass spectrometry. Mol Cell Proteomics 2007;6:1088-102.

[26] Solito E, de Coupade C, Parente L, Flower RJ, Russo-Marie F. IL-6 stimulates annexin 1 expression and translocation and suggests a new biological role as class II acute phase protein. Cytokine 1998;10:514-21.

[27] Solito E, de Coupade C, Parente L, Flower RJ, Russo-Marie F. Human annexin 1 is highly expressed during the differentiation of the epithelial cell line A 549: involvement of nuclear factor interleukin 6 in phorbol ester induction of annexin 1. Cell Growth Differ 1998;9:327-36

[28] Nakashima Y, Fujii H, Sumiyoshi S, Wight TN, Sueishi K. Early human atherosclerosis: accumulation of lipid and proteoglycans in intimal thickenings followed by macrophage infiltration. Arterioscler Thromb Vasc Biol 2007;27:1159-65.

[29] Majesky MW. Developmental basis of vascular smooth muscle diversity. Arterioscler Thromb Vasc Biol 2007;27:1248-58.

[30] Owens GK, Kumar MS, Wamhoff BR. Molecular regulation of vascular smooth muscle cell differentiation in development and disease. Physiol Rev 2004;84:767-801.

[31] Jovinge S, Hultgardh-Nilsson A, Regnstrom J, Nilsson J. Tumor necrosis factor-alpha activates smooth muscle cell migration in culture and is expressed in the ballooninjured rat aorta. Arterioscler Thromb Vasc Biol 1997;17:490-7. 